\title{
A digital intervention targeting cognitive control network dysfunction in middle age and older adults with major depression
}

\author{
Faith M. Gunning (10), Joaquin A. Anguera ${ }^{2}$, Lindsay W. Victoria ${ }^{1}$ and Patricia A. Areán ${ }^{3}$
}

\begin{abstract}
Nonpharmacological interventions targeting putative network mechanisms of major depressive disorder (MDD) may represent novel treatments. This mechanistic study investigates how a video game-like intervention, designed to improve cognitive control network (CCN) functioning by targeting multitasking, influences the CCN of middle-aged and older adults with MDD. The sample consisted of 34 adults aged 45-75 with SCID-defined diagnosis of MDD, Hamilton depression rating scale scores $\geq 20$, and a deficit in cognitive control. Participants were instructed to play at home for 20-25 min per day, at least 5 times per week, for 4 weeks. Evidence of target engagement was defined a priori as $>2 / 3$ of participants showing CCN improvement. CCN engagement was defined as a change in a Z score of $\geq 0.5$ on functional magnetic resonance imaging (fMRI) in activation and functional connectivity of the CCN during task-based and resting-state $\mathrm{fMRI}$, respectively. $74 \%$ of participants showed a change in activation of the CCN, and $72 \%$ showed an increase in resting-state functional connectivity. Sixty-eight percent demonstrated improved cognitive control function, measured as either improvement on sustained attention or working memory performance or reduced self-reported symptoms of apathy on the frontal systems behavioral scale (FrsBe). Participants also reported a significant reduction in mood symptoms measured by PHQ-9. A remotely deployed neuroscience-informed video game-like intervention improves both CCN functions and mood in middle-aged and older adults with MDD. This easily-disseminated intervention may rescue CCN dysfunction present in a substantial subset of middle-aged and older adults with MDD.
\end{abstract}

\section{Introduction}

At least one-third of individuals treated with antidepressant medications or psychotherapy do not achieve adequate response, even after multiple treatments trials ${ }^{1-3}$. Even when standard treatments improve mood symptoms, many individuals, especially older adults, are left with persistent executive dysfunction ${ }^{4-7}$ that is associated with disability $^{5-7}$, and increased risk of depression recurrence ${ }^{5-9}$. Thus far, alternative pharmacologic approaches designed to target comorbid executive dysfunction demonstrate limited

\footnotetext{
Correspondence: Faith M. Gunning (fgd2002@med.cornell.edu) ${ }^{1}$ Department of Psychiatry, Weill Cornell Medicine, New York, NY, USA ${ }^{2}$ Departments of Neurology and Psychiatry, University of California San Francisco, San Francisco, CA, USA

Full list of author information is available at the end of the article
}

efficacy $^{8,10}$. Likewise, psychotherapy we optimized to treat individuals suffering from major depressive disorder (MDD) with executive dysfunction improved mood symptoms $^{11,12}$, but not executive dysfunction ${ }^{13}$. Novel strategies are needed that target persistent dysfunction among individuals with MDD.

Executive dysfunction present in older individuals with MDD appears to reflect inefficient functioning of the cognitive control network $(\mathrm{CCN})^{14-18}$. The CCN, comprised of the dorsal anterior cingulate cortex (dACC), dorsolateral prefrontal cortices, and posterior parietal cortices support the flexible maintenance of goal-directed behavior in the face of changing internal and environmental demands ${ }^{19,20}$. Nonpharmacological interventions that aim to rescue $\mathrm{CCN}$ network dysfunctions could 
therefore represent a promising, personalized approach for the large subset of individuals with MDD who have an inefficient $\mathrm{CCN}$ and are often less likely to benefit from standard treatments.

One method to target $\mathrm{CCN}$ dysfunction applies digital cognitive interventions designed to improve $\mathrm{CCN}$ functions through the repetitive engagement of the network. We conducted a preliminary, proof of concept RCT of a video game-like intervention designed to target the $\mathrm{CCN}$ by improving age-related deficits in multitasking ${ }^{13}$. In that study, individuals over the age of 60 in a major depressive episode were randomized to problem-solving therapy (PST), efficacious psychotherapy for older adults with depression, or digital intervention. Although the two groups showed a similar improvement in depression, only the group randomized to the multitasking game-like condition showed significant improvement in working memory and sustained attention performance ${ }^{13}$.

The results of this pilot RCT shed limited light on the mechanism of action of the intervention. In fact, nearly all non-pharmacologic intervention studies in MDD focus on demonstrating efficacy, without confirming target engagement ${ }^{21}$. That is they may show clinical benefit without demonstrating how an intervention engages the hypothesized target. In the present study, we aimed to determine whether an algorithmically delivered video game-like intervention targeting multitasking can modulate $\mathrm{CCN}$ function in middle-aged and older adults with MDD. We hypothesized that after 4 weeks of training with this intervention at least $2 / 3$ of participants would show a significant improvement in focal activation of the anterior $\mathrm{CCN}$ and an increase in functional connectivity of the $\mathrm{CCN}$ at rest. In addition, we assessed whether improvement in $\mathrm{CCN}$ functioning would be reflected in a similar degree and frequency of improvement demonstrated on either measure of cognitive control performance as assessed by a visual continuous performance task (CPT) and a delayed recognition working memory task or decreased complaints on the apathy subscale of the frontal systems behavior scale (FrSBe $)^{22}$.

\section{Materials and methods \\ Study design}

This study was the proof-of-concept phase of a twophase clinical trial to evaluate mechanisms of action of the video game-like intervention, specifically engagement of $\mathrm{CCN}$ as measured by functional magnetic resonance imaging (fMRI), computerized sustained attention, and working memory tasks, and self-reported symptoms of apathy. The study used a single-arm, open-label design. Measures of $\mathrm{CCN}$ target engagement were administered at baseline/pre-intervention and week 4/post-intervention with analysis that focused on changes in $\mathrm{CCN}$ function over 4 weeks.

\section{Participants}

Participants were adults aged 45-75. Prior to study entry, MDD diagnosis was confirmed by Structured Clinical Interview for DSM-V $(\mathrm{SCID})^{23}$, conducted by a study clinician. Eligibility required baseline Hamilton depression rating $\mathrm{scale}^{24}$ scores $\geq 20$ (i.e., moderate severity). Participants were required to demonstrate a deficit in cognitive control, defined as $>1$ standard deviation (SD) below the mean for their age and education on at least one of the following: Stroop Color Word Interference ${ }^{25}$, Trail Making Part $\mathrm{B}^{26}$, or FrSBe ${ }^{22}$. Participants were either not currently on antidepressants or on a stable dose for at least 12 weeks, with no intent to change dose during study participation. Individuals were excluded if they had a mini-mental state examination $(\mathrm{MMSE})^{27}$ score $>1$ SD below the mean for their age and education level, or a contraindication to MRI. Individuals with a history of neurologic illnesses, psychosis or mania, or acute medical illnesses were also excluded.

All participants provided written informed consent, as per the study protocol approved by the IRBs of Weill Cornell Medicine, University of Washington, and the Nathan Kline Institute.

\section{Intervention}

The intervention is a self-guided therapeutic video game-like intervention (Project: Evolution [EVO], Akili Interactive Labs, Cambridge, MA) that involves a combination of visuomotor and perceptual discrimination tasks intended to adaptively treat multi-tasking deficiencies $^{28}$. The game begins with an assessment of the participants' multi-tasking cost, i.e., tests of reaction time alone, navigation alone, and then the two domains combined. This sets the level of difficulty for each participant, personalizing the training experience. Improvement throughout the intervention transports the participant to different visual "worlds", designed to immerse the player and enhance the depth of engagement and compliance. Frequent performance assessments are embedded in the program to determine the level of improvement during training and adaptively set a therapeutic regimen based on the participant's performance. Thus, the participant is challenged to continue to improve upon their own performance to reach the next level. The same adaptive mechanics utilized in the assessment are employed in the training sessions which keep the player at $\sim 80 \%$ accuracy.

The intervention relied on at-home use of the therapeutic video game, delivered via Apple iPad, and 4 weekly in-person visits with a Masters or PhD-level care manager who provided instruction and technical support for participation in the intervention. At each study visit the participants completed the patient health questionnaire-9 (PHQ-9) ${ }^{29}$ to assess depressive symptoms as part of safety and mood monitoring. 
During the first session, care managers introduced the game, observed participants' completion of a practice session, and set an action plan with participants to ensure regular gameplay. During the weekly follow-up sessions, care managers reviewed the adherence and action plan and a dashboard within the application tracking performance and time played. They worked with participants to adjust the action plan as needed if adherence was low, defined as less than 5 days of gameplay.

The intervention required 20-25 min of gameplay for a minimum of 5 days and up to 7 days per week. The game locked after five sessions per day.

\section{Measures of target engagement}

The MRI, computerized cognitive control tasks, and self-report measures of $\mathrm{CCN}$ function were collected at baseline and following 4 weeks of the intervention.

\section{Circuitry}

CCN circuitry was measured by assessing focal activation of the anterior CCN using task-based fMRI during a Stroop/Flanker task and resting-state functional connectivity (rsFC) of the CCN. Details related to scanning parameters, image acquisition, and preprocessing steps are described in the Supplementary Information.

\section{Stroop/flanker task}

Participants viewed three color words on the screen: the central congruent or incongruent Stroop ${ }^{25}$ target word and two surrounding Flanker ${ }^{30}$ words. They responded to the ink color of the central target word with a button press while ignoring multiple sources of cognitive interference: what the word says and the surrounding distractor word. The main contrast of interest for the task-based MRI analyses focused on the congruency of the Stroop targets: congruent (e.g., "purple" in purple ink) vs. incongruent (e.g., "purple" in orange ink). See Supplementary Fig. 1 for more details.

Following preprocessing of the task-based data, we extracted percent signal activation during the Stroop/ Flanker task in regions of the anterior portion of the $\mathrm{CCN}$ : middle frontal gyrus (MFG) and AACC from baseline to week 4 MRI. Beta values were extracted for incongruent trials only since they had the highest cognitive control load. Our z-scored measure of target engagement for task-based activation was calculated as follows: (week 4 incongruent MFG and dACC activation - baseline MFG and dACC activation)/baseline SD of MFG and dACC activation. The mean and SD of the extracted beta values were calculated within-region.

\section{rsFC}

We conducted a seed-based rsFC analysis to investigate the change in connectivity among nodes of the anterior portion of the $\mathrm{CCN}$ (i.e., MFG and $\mathrm{dACC}$ ) following 4 weeks of the intervention.

To examine target engagement, we measured within$\mathrm{CCN}$ connectivity between MFG and dACC. Following preprocessing of the resting-state data, we extracted correlation coefficients between these regions at baseline and week 4 of EVO with $4 \mathrm{~mm}$ spherical seeds placed bilaterally in the MFG (MNI coordinates - 36/36, 28, 34) and the dACC (MNI coordinates $-4 / 4,30,22$ ). Our zscored measure of target engagement for the rsFC analysis was calculated as follows: (week 4 partial correlation between MFG and AACC - baseline partial correlation between MFG and dACC)/baseline SD of partial correlation between MFG and dACC.

\section{Cognitive control performance}

Two cognitive tasks were used to measure target engagement of the $\mathrm{CCN}$ : (1) a delayed recognition working memory task (called "AID" for attending or ignore distractor $)^{31}$; (2) a continuous performance test $(\mathrm{CPT})^{32}$, a measure of sustained attention.

\section{AID}

The AID includes aspects of visual information processing that are held constant ("Is the final face shown the same as the first?") while other task demands are manipulated. Our analysis focused on the "ignore distractor" (ID) condition, during which participants are instructed to ignore the distracting face while maintaining the representation of the cue face. We analyzed RT during this condition ${ }^{13,28}$ and calculated Z-scores as follows: (week 4 ignore distractor RT - baseline ignore distractor RT)/baseline SD of ignoring distractor RT.

\section{CPT}

We used a $\mathrm{CPT}^{32}$, a 23-min, fixed interval, visual CPT that we modeled after the test of variable attention (TOVA $)^{33}$. Participants were instructed to respond to the target (white square at the top edge of the screen) and to ignore the non-target (white square at the bottom edge of the screen). We analyzed response time in the sustained attention condition (target stimuli appears in $22 \%$ of trails) and calculated $Z$-scores as follows: (week 4 sustained attention RT - baseline sustained attention RT)/ baseline SD of sustained attention RT.

\section{Self-reported cognitive control}

We used the apathy subscale of the $\mathrm{FrSBe}^{22}$, a selfreport scale that measures executive function. We examined the apathy subscale to capture cognitive symptoms that are likely not represented in the other cognitive measures. We analyzed the FrSBe apathy subscale and calculated z-scores as follows: (week 4 score baseline score)/baseline SD of the score. 


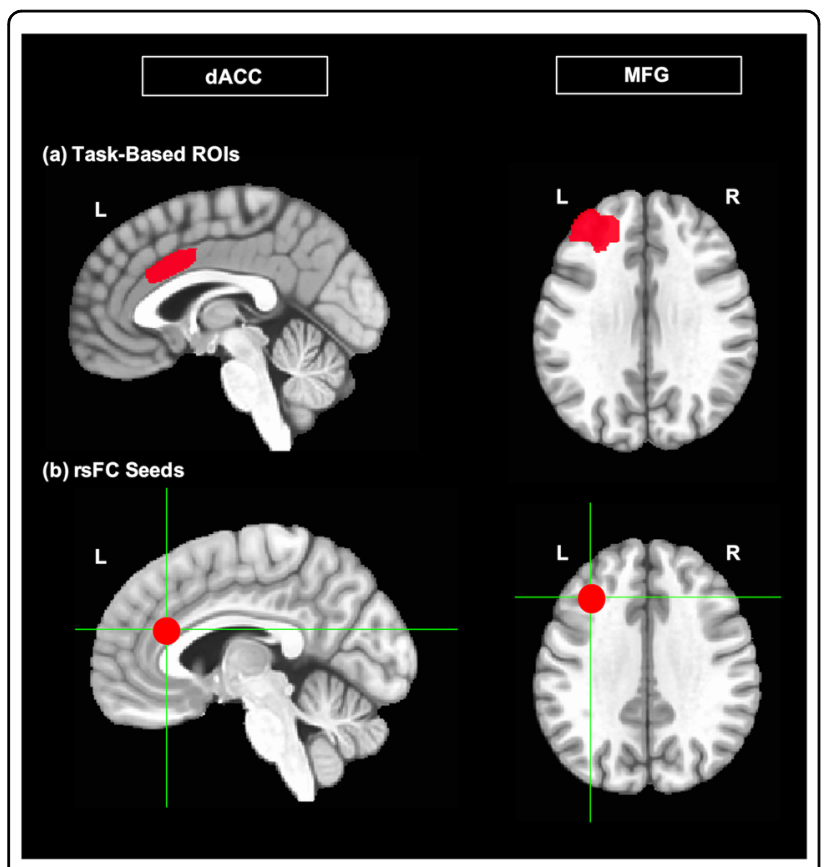

Fig. $1 \mathrm{~A}$ priori regions of interest (ROIs) in the cognitive control network (CCN) defined for circuitry-based analysis: (left) middle frontal gyrus (MFG); (right) dorsal anterior cingulate cortex (dACC). a ROls from which beta values were extracted for Stroop/ Flanker task-based analysis of target engagement. $\mathbf{b}$ Placement of $4 \mathrm{~mm}$ spherical seeds in the MFG and dACC for resting-state functional connectivity ( $\mathrm{rFF}$ ) analysis of target engagement.

\section{Data analysis and go/no-go decision}

The primary purpose of this phase of the clinical trial was to establish whether or not the intervention engaged the hypothesized $\mathrm{CCN}$ target. We defined the go/no-go criteria a priori, informed by other proof-ofconcept trial methodology 34,35 . We determined that $66 \%$ of the sample would need to show significant improvement in the CCN on fMRI (task-based activation and rsFC) and either CCN task performance or self-reported symptoms of cognitive control. Significant improvement was defined as Z-scores $\geq 0.5$ for pre- to post-EVO change in $\mathrm{CCN}$ task-based activation and functional connectivity, as well as CPT and/or AID performance, or FrSBe self-report score.

Secondary analyses of CCN circuitry included exploratory whole-brain analyses of Stroop/Flanker task performance pre- and post-EVO, as well as whole-brain seed-based exploration of changes in rsFC from pre- to post-EVO. The methodological details of these analyses are described in Supplementary Information. We also explored the association between changes in rsFC and post-intervention performance and self-reported CCN function with correlational analyses comparing the Z-scored change in rsFC pre- to post-EVO to post-EVO performance on the CPT
Table 1 Proportion of participants meeting target engagement goal for each level of analysis of cognitive control function.

\begin{tabular}{ll}
\hline Level of analysis & $\boldsymbol{N}(\%)$ \\
\hline Cognitive control performance: AID/CPT & $23 / 34(68 \%)$ \\
Self-reported cognitive control symptoms: FrSBe & $23 / 34(68 \%)$ \\
CCN circuitry (task-based activation) & $25 / 34(74 \%)$ \\
CCN circuitry (rsFC) & $21 / 29(72 \%)$ \\
\hline
\end{tabular}

CPT continuous performance task, FrSBe frontal systems behavior scale, CCN cognitive control network, $r s F C$ resting-state functional connectivity.

The successful target engagement goal was defined as $66 \%$ of participants with significant improvement ( $z$-score $\geq 0.5$ ) in CCN measures (performance, selfreport, circuitry) following 4 weeks of EVO.

and AID tasks and post-EVO self-reported FrSBe scores. These results are reported as Pearson's correlation coefficients.

Exploratory analysis of depression outcomes consisted of repeated measures ANOVA to determine changes in mood over time, the proportion of participants exhibiting response (defined as a 50\% decrease in PHQ-9 scores from baseline to 4 weeks), and remission (defined as a PHQ-9 score of less than 5 at 4 weeks).

\section{Results}

\section{Sample characteristics}

Forty-one individuals were enrolled in the trial. Seven participants were excluded from the analysis (see Supplementary Fig. 2 for participant flow diagram), yielding a final sample of 34 participants. Seventy-one percent $(N=$ $24)$ of the sample was female, aged $45-75$ years $(M=61.6$ years, $\mathrm{SD}=9.4)$. The mean education level was 15.8 years $(\mathrm{SD}=2.2)$ and the mean MMSE score was $28.9(\mathrm{SD}=$ 1.0). At the baseline visit, the mean HAM-D score was 24.4 $(\mathrm{SD}=4.9)$. All subjects completed 4 weeks of the intervention.

\section{Target engagement \\ CCN circuity (task-based activation)}

To assess activation, beta values were extracted from AFNI Daemon atlas-based ROIs in the MFG and dACC (Fig. 1a). Twenty-one of the 34 participants (74\%) showed greater change in activation of the CCN (MFG and/or $\mathrm{dACC}$ ) for incongruent trials (i.e., trials with a higher cognitive control load) after 4 weeks of EVO (Table 1).

\section{CCN circuitry (rsFC)}

Five participants were excluded from the rsFC analysis due to a high degree of motion in the resting-state scan (see Supplementary Information for details of the motion parameters for the rsFC analysis). To assess connectivity, we extracted correlation coefficients between MFG and dACC seeds (Fig. 1b) at baseline and week 4 of the intervention. 21 

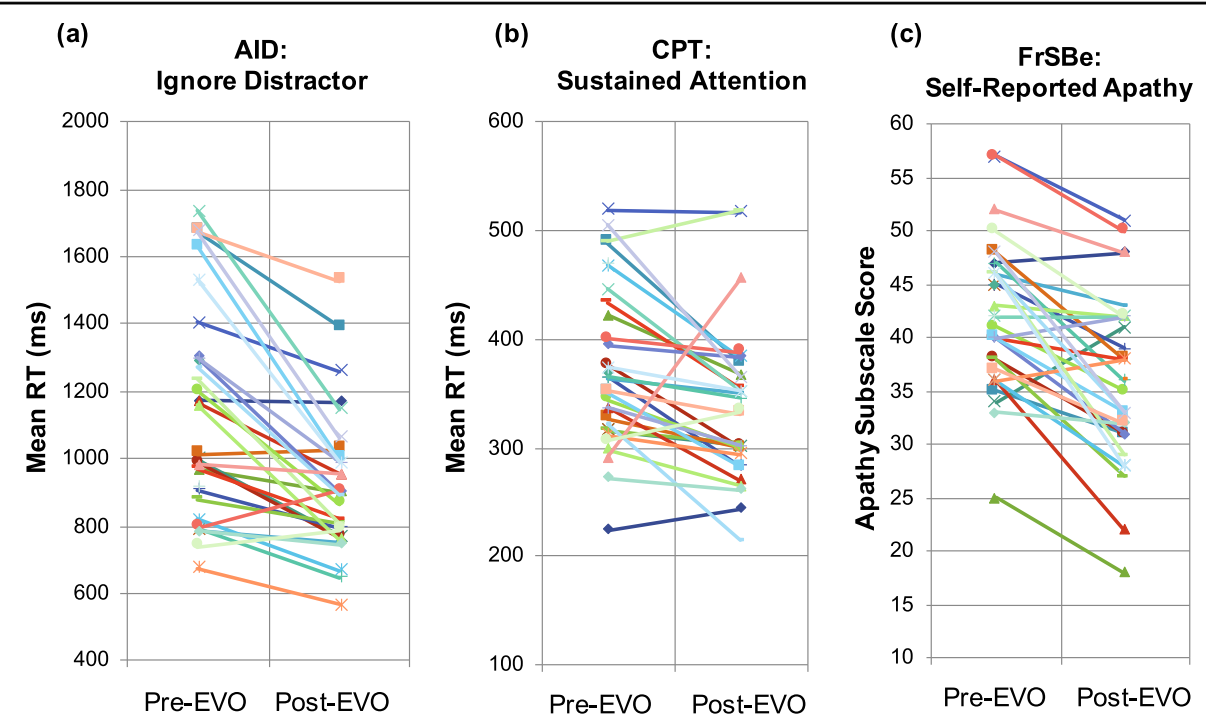

Fig. 2 Improvement in cognitive control performance and self-reported cognitive control symptoms following 4 weeks of the EVO intervention. a Change in mean response time (RT) from pre- to post-EVO on the lgnore Distractor condition of the AID task, a measure of working memory. (Pre-EVO: $M=1141.50 \mathrm{~ms}$ (321.23); post-EVO: $M=920.98 \mathrm{~ms}$ (214.39); pre vs. Post: $\mathrm{t}(29)=5.92, p<0.001$ ). b Change in mean RT from pre- to post-EVO on the Sustained Attention condition of the CPT. (Pre-EVO: $M=1069.38$ ms (261.25); Post-EVO: $M=886.67$ ms (204.19); pre vs. Post: $t(29)=$ $5.00, p<0.001)$. $c$ Change in score from pre- to post-EVO on the Apathy Subscale score of the FrSBe self-report scale. (Pre-EVO: M = 42.30 (7.13); PostEVO: $M=36.03$ (8.01); pre vs. post: $\mathrm{t}(29)=5.82, p<0.001$ ).

of the 29 participants (72\%) showed an increase in rsFC between seeded regions of the CCN after 4 weeks of EVO.

\section{Cognitive control performance (AID/CPT)}

Twenty-three of the 34 participants $(68 \%)$ showed an improvement $\geq 0.5$ on our composite Z-score of CCN performance (on the AID and/or the CPT task) after 4 weeks of video game use. Participants were significantly faster from pre- to post-intervention on the ignore distractor condition of the AID and the sustained attention condition of the CPT (Fig. 2a, b).

\section{Self-reported cognitive control symptoms (FrSBe)}

Twenty-three of the 34 participants $(68 \%)$ showed an improvement $\geq 0.5$ on our composite $Z$-score of selfreported $\mathrm{CCN}$ symptoms (on the AID and/or the CPT task) after 4 weeks of EVO. Self-reported apathy was significantly reduced pre- to post-intervention (Fig. 2c).

For exploratory subgroup analyses examining relationships of gender, age, and duration of gameplay to target engagement, see Supplementary Table 1.

\section{Secondary analyses}

\section{Whole-brain fMRI analysis}

Task-based activation following 4 weeks of therapeutic video game use

To explore the pattern of whole-brain activation following use of the intervention, we assessed activation of incongruent relative to congruent Stroop/Flanker trials A group contrast map showing all incongruent trials minus all congruent (collapsed across Flanker similarity) trials at week 4 revealed a positive cluster (more activation for incongruent than for congruent trials) surviving cluster correction, located in the right MFG (cluster size $=39$ voxels; Peak coordinates: 50, 21, 30.5; Fig. 3a). Participants also demonstrated improved performance on the Stroop/Flanker task after 4 weeks of EVO (see Supplementary Fig. 3).

Resting-state connectivity following 4 weeks of therapeutic video game use

Increased rsFC following EVO was observed between the left MFG seed and a cluster located contralaterally in the right MFG (cluster size $=50$ voxels; peak MNI coordinates: $30,48,27$; peak intensity $=5.4174$ ). Increased rsFC following EVO was also observed between the right MFG seed and two clusters: one located in the right inferior frontal gyrus (IFG) (cluster size $=43$ voxels; peak MNI coordinates: 42,24 , 9; peak intensity $=4.4314$ ) and one located in the right superior temporal gyrus (STG) (cluster size $=95$ voxels; peak MNI coordinates: $54,-36,15$; peak intensity $=5.0816$ ). Significant clusters are displayed in Fig. 3b.

Improvement in CCN circuitry with therapeutic video game use is associated with post-intervention cognitive control performance and self-reported apathy

We observed change in rsFC of the $\mathrm{CCN}$ following 4 weeks of video gameplay was associated with postintervention scores on behavioral and self-report 


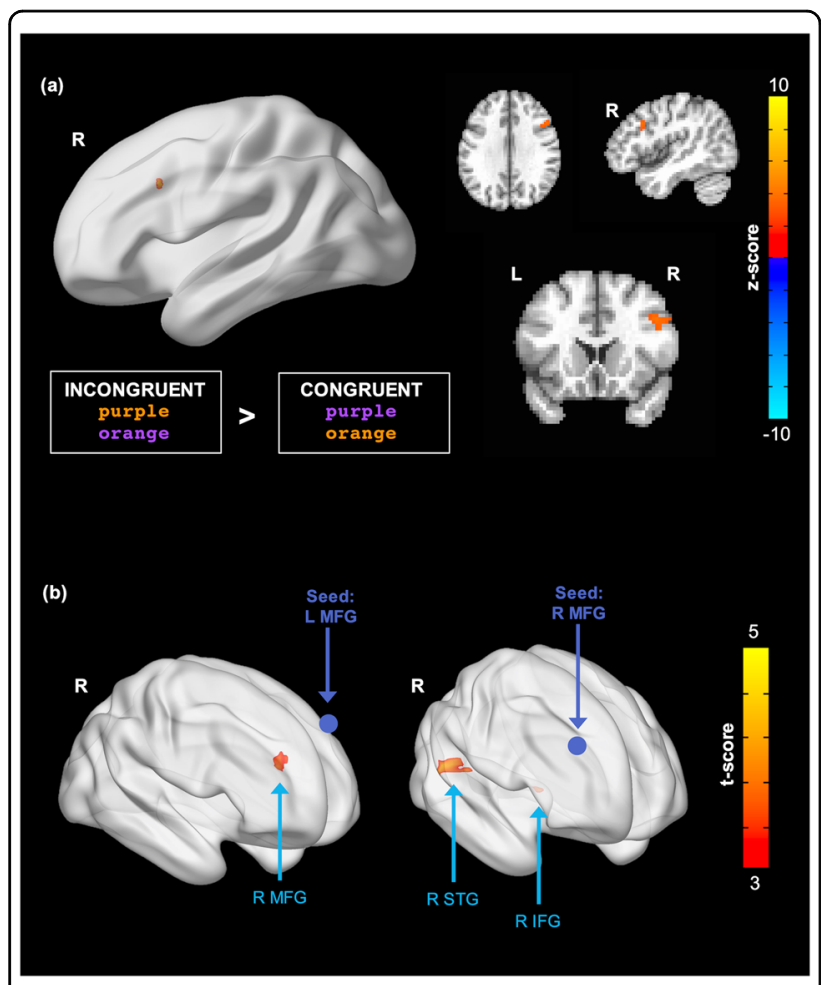

Fig. 3 Exploratory whole-brain analysis of changes in task-based activation and resting-state functional connectivity (rsFC)

following $\mathbf{4}$ weeks of the intervention. a Greater activation at postEVO in the right middle frontal gyrus (MFG) during the Stroop/Flanker task for incongruent trials relative to congruent trials; voxel threshold: $p<0.005$, cluster threshold: $p<0.07$. b Increased functional

connectivity from pre- to post-EVO from the left MFG seed to the right MFG and the right MFG seed to the right superior temporal gyrus (STG) and right inferior frontal gyrus (IFG); voxel threshold: $p<0.001$, cluster threshold: $p<0.05$.

measures of CCN target engagement. Specifically, pre- to post-EVO change in rsFC between R MFG and R STG was associated with greater working memory ability (measured by AID performance in the "ignore distractor" condition) post-intervention $(r(23)=0.44, p=0.03$; Fig. $4 \mathrm{a})$, but was not significantly correlated with sustained attention (measured by CPT performance) postintervention $(r(24)=-0.22, p=0.28$; Fig. $4 \mathrm{~b})$. Pre- to post-EVO change in rsFC between R MFG and R STG was associated with reduced self-reported apathy (measured by FrSBe self-report score) post-intervention $(r(24)=-0.44, p=0.02$; Fig. $4 \mathrm{c})$.

\section{Exploratory analysis}

Improvement in self-reported mood symptoms over time: we observed a significant improvement in depression severity over time, as measured by PHQ-9 score (by repeated-measures ANOVA, $\mathrm{F}[4,25]=16.5, p<0.001$; Supplementary Fig. 4). Among 32 individuals with baseline and week 4 PHQ-9, 13 (41\%) met the criteria for treatment response and 7 (22\%) for remission.

\section{Discussion}

In this investigation of a neuroscience-informed therapeutic video game-like intervention for MDD, we confirmed the engagement of $\mathrm{CCN}$ using brain-based measures (task-based fMRI, resting-state fMRI), sustained attention or working memory performance (CPT, AID), and self-reported behavioral symptoms of cognitive control disturbance (Apathy subscale of the FrsBE). Specifically, we found that a digital therapeutic strategy designed to improve cognitive control abilities (i.e., multitasking) improved brain-based focal activation and rsFC functional connectivity on fMRI of the $\mathrm{CCN}$ in more than $70 \%$ of our sample of middle-aged and older adults with MDD and cognitive control dysfunction. In parallel, we identified significant improvements in performance and selfreported CCN functions in $68 \%$ of participants. Taken together, these convergent results demonstrate target engagement of the $\mathrm{CCN}$.

These findings extend our prior proof-of-concept randomized controlled trial conducted in an elderly sample of individuals suffering from a major depressive episode. That trial demonstrated similar antidepressant efficacy for the game intervention and PST, but only the former was associated with improvement in cognitive control performance ${ }^{13}$. However, that study could not address the mechanism of action. Here, we demonstrate that improvement in executive functions and depression may be the results of a change in activation and functional connectivity of $\mathrm{CCN}$, validating this novel treatment target.

As in the prior study, our exploratory analyses found this gamified intervention to have a significant impact on mood after 4 weeks of treatment. More than half of those treated met the criteria for symptomatic response, and one quarter for remission. Notably, this is a sample characterized with risk factors for poor response to antidepressant treatment ${ }^{7,36-40}$, in which response rates after 8 weeks of antidepressant medication treatment have been reported to be between $25 \%$ and $60 \%^{7,40}$.

Our study adds to a growing literature indicating that gamified interventions may impact depressive symptoms $^{41-44}$. Recent meta-analyses of prior cognitive training interventions showed a small to moderate effect size for symptom improvement (0.43) in depression ${ }^{45}$. The majority of these trials have been relatively small and, with few exceptions ${ }^{42,43}$, have not directly interrogated the underlying neural mechanisms tied to improved mood-based symptoms. In this study, the intervention was designed to selectively improve $\mathrm{CCN}$ functions that are both susceptible to effects of normal aging and predictive of poor antidepressant response in 
(a)

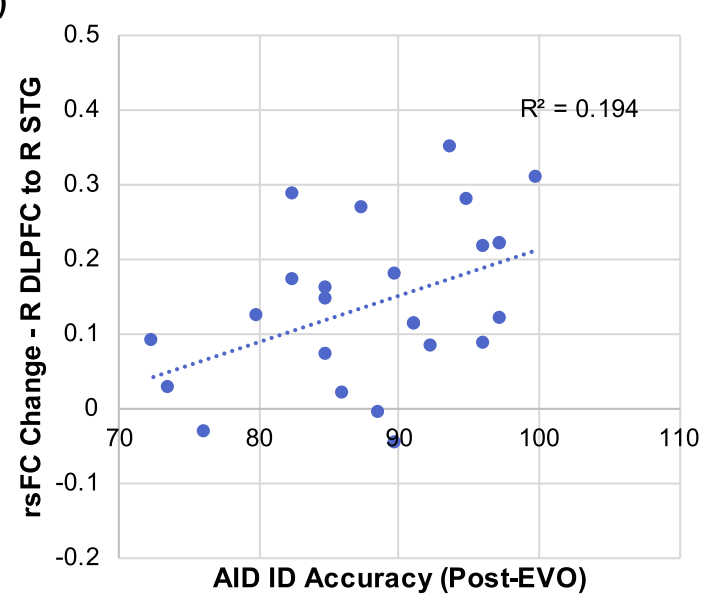

(b)

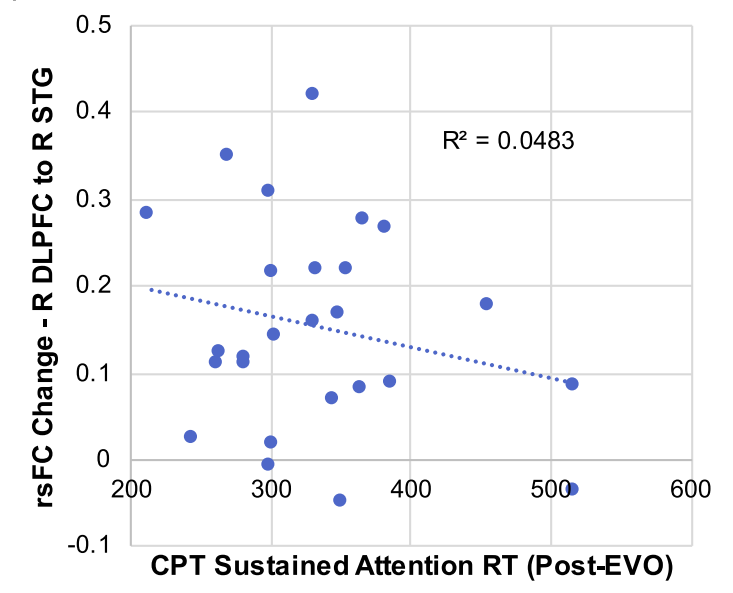

(c)

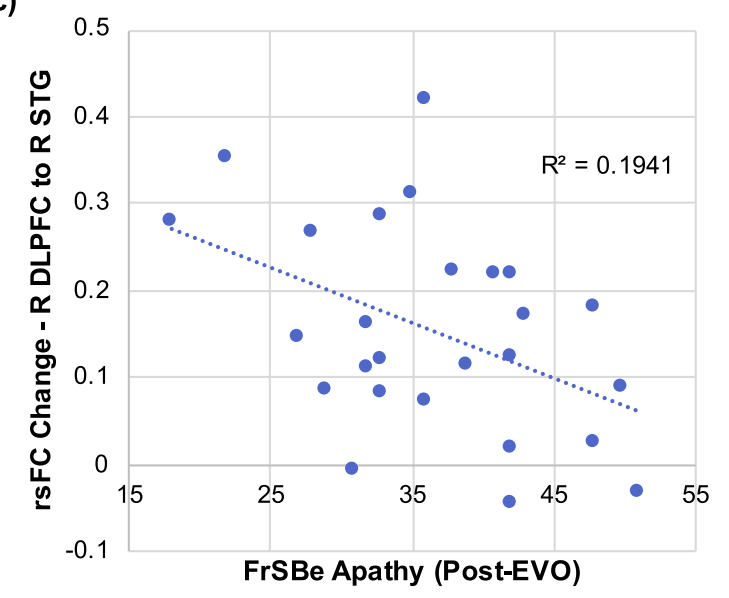

Fig. 4 Association between pre- to post-EVO change in CCN rsFC is and cognitive control performance and self-reported symptoms post-EVO. a Change in rsFC between the R MFG and R STG is significantly correlated with accuracy on the "Ignore distractor" condition of the AID task post-EVO. $\mathbf{b}$ Change in rsFC between the R MFG and R STG is significantly not correlated with performance on the "Sustained

Attention" condition of the CPT post-EVO. $c$ Change in rsFC between the $R$ MFG and R STG is significantly correlated with post-intervention scores on the Apathy Subscale score of the FrSBe self-report scale. older individuals with MDD. The current findings, in conjunction with our previous results, suggest that the engagement of the $\mathrm{CCN}$ through repeated cognitive stimulation may be a central mechanism for rescuing dysfunctional brain networks that contribute to both the mood and cognitive symptoms in many suffering from MDD.

We note several caveats. Although we demonstrate significant changes in $\mathrm{CCN}$ functions using converging methodologies, without a control group we cannot state with confidence that these changes are attributable to the intervention until it is compared to an active, equally engaging control. Further, the modest sample size requires replication of the target engagement findings. Likewise, changes in mood could be a function of regularly engaging in an entertaining activity or receiving weekly visits with a care manager. Participants completed the AID and CPT tasks at baseline and week 4 of the study so practice effects may account for some of the improvement in performance following treatment. We also cannot assess the specificity of effecti.e., whether the similar benefit would be observed in individuals with MDD but without executive dysfunction. It is possible that only those with dysfunction in the $\mathrm{CCN}$ will benefit from an intervention designed to target this deficit ${ }^{46}$, whereas this approach may not be effective for individuals whose depression may be mediated by deficits in other brain networks ${ }^{47}$. Preliminary analyses suggest age and gender may be potential moderators of target engagement. However, In light of the modest sample size and risk for type 1 and type 2 error, such associations require confirmation in larger studies.

\section{Conclusions}

This study suggests that a remotely deployed neuroscience-informed video game-like intervention improves both CCN functions and mood in middle-aged and older adults with MDD. This phase of a two-phase clinical trial focused on the ability of a video game-like intervention designed to improve multitasking to engage the CCN. The next steps require confirmation of engagement of the $\mathrm{CCN}$ target in a randomized trial that includes an expectancy-matched video game control played at the same frequency and duration as the experimental intervention. Subsequent investigation in a larger cohort will also be required to identify whether particular patient subgroups benefit differentially in terms of both CCN function and mood symptoms. Nevertheless, this study underscores the importance of the $\mathrm{CCN}$ as a target for interventions aimed at the treatment of MDD, addressing symptoms that are frequently resistant to traditional antidepressant treatments in mid-to-late adulthood. 


\section{Acknowledgements}

This study was funded by the National Institute of Mental Health under award number MH1 10509. Project: Evolution [EVO] and Pads for participants were supplied by Akili Interactive; no financial support was provided by the company. We thank Dr. Mathew Hoptman and Dr. Matteo Respino for their roles in the choice of MRI scan acquisition parameters and preprocessing of MRI data, respectively. In addition, we thank Dr. Mathew Hoptman and the staff of the Center for Biomedical Imaging and Neuromodulation of The Nathan Kline Institute for Psychiatric Research where MRI data were acquired.

\section{Author details}

'Department of Psychiatry, Weill Cornell Medicine, New York, NY, USA. ${ }^{2}$ Departments of Neurology and Psychiatry, University of California San Francisco, San Francisco, CA, USA. ${ }^{3}$ Department of Psychiatry and Behavioral Sciences, University of Washington, Seattle, WA, USA

\section{Conflict of interest}

The authors declare no competing interests.

\section{Publisher's note}

Springer Nature remains neutral with regard to jurisdictional claims in published maps and institutional affiliations.

Supplementary information The online version contains supplementary material available at https://doi.org/10.1038/s41398-021-01386-8.

Received: 1 December 2020 Revised: 8 April 2021 Accepted: 20 April 2021 Published online: 04 May 2021

\section{References}

1. Gaynes, B. N. et al. What did STAR*D teach us? Results from a large-scale, practical, clinical trial for patients with depression. Psychiatr. Sen. 60, 1439-1445 (2009).

2. Insel, T. R. \& Wang, P. S. The STAR*D trial: revealing the need for better treatments. Psychiatr. Serv. 60, 1466-1467 (2009).

3. Cuijpers, P. et al. The efficacy of psychotherapy and pharmacotherapy in treating depressive and anxiety disorders: a meta-analysis of direct comparisons. World Psychiatry 12, 137-148 (2013).

4. Zuckerman $\mathrm{H}$. et al. Recognition and treatment of cognitive dysfunction in major depressive disorder. Front. Psychiatry 9 (2018).

5. McIntyre, R. S. et al. Cognitive deficits and functional outcomes in major depressive disorder: determinants, substrates, and treatment interventions. Depress. Anxiety 30, 515-527 (2013).

6. Kiosses, D. N. \& Alexopoulos, G. S. IADL functions, cognitive deficits, and severity of depression: a preliminary study. Am. J. Geriatr. Psychiatry 13, 244-249 (2005).

7. Alexopoulos, G. S. et al. Executive dysfunction and the course of geriatric depression. Biol. Psychiatry 58, 204-210 (2005).

8. Alexopoulos, G. S. Mechanisms and treatment of late-life depression. Transl. Psychiatry 9, 1-6 (2019).

9. Rock, P. L., Roiser, J. P., Riedel, W. J. \& Blackwell, A. D. Cognitive impairment in depression: a systematic review and meta-analysis. Psychol. Med. 44, 2029-2040 (2014).

10. Pan, Z. et al. Pharmacological treatment of cognitive symptoms in major depressive disorder. CNS Neurol. Disord. 16, 1-9 (2017).

11. Areán, P. A. et al. Problem-solving therapy and supportive therapy in older adults with major depression and executive dysfunction. Am. J. Psychiatry 167, 1391-1398 (2010).

12. Alexopoulos, G. S. et al. Problem-solving therapy and supportive therapy in older adults with major depression and executive dysfunction: effect on disability. Arch. Gen. Psychiatry 68, 33-41 (2011).

13. Anguera, J. A., Gunning, F. M. \& Areán, P. A. Improving late life depression and cognitive control through the use of therapeutic video game technology: a proof-of-concept randomized trial. J. Depress Anxiety 34, 508-517 (2017). doi:
14. Gandelman, J. A. et al. Intrinsic functional network connectivity is associated with clinical symptoms and cognition in late-life depression. Biol. Psychiatry Cogn. Neurosci. Neuroimaging 4, 160-170 (2019).

15. Alexopoulos, G. S. et al. Functional connectivity in the cognitive control network and the default mode network in late-life depression. J. Affect Disord. 139, 56-65 (2012).

16. Respino, M. et al. Cognitive control network homogeneity and executive functions in late-life depression. Biol. Psychiatry Cogn. Neurosci. Neuroimaging 5, 213-221 (2020)

17. Respino, M. et al. The impact of white matter hyperintensities on the structural connectome in late-life depression: relationship to executive functions. Neurolmage 6, 654-666 (2019).

18. Tadayonnejad, R. \& Ajilore, O. Brain network dysfunction in late-life depression: a literature review. J. Geriatr. Psychiatry Neurol. 27, 5-12 (2014).

19. Niendam, T. A. et al. Meta-analytic evidence for a superordinate cognitive control network subsenving diverse executive functions. Cogn. Affect Behav. Neurosci. 12, 241-268 (2012).

20. Cole, M. W. \& Schneider, W. The cognitive control network: integrated cortical regions with dissociable functions. Neuroimage 37, 343-360 (2007).

21. Insel, T. R. The NIMH experimental medicine initiative. World Psychiatry 14, 151-153 (2015).

22. Grace, J., \& Malloy, P. F. Frontal systems behavior scale (FrSBe): professional manual. Lutz, FL: Psychol. Assess. Resour. (PAR) (2001).

23. First M. Williams J., Karg R., Spitzer R. Structured Clinical Interview for DSM-5Research Version (SCID-5 for DSM-5, Research Version; SCID-5-RV). Arlington, VA: American Psychiatric Association; 2015: 1-94.

24. Hamilton, M. A rating scale for depression. J. Neurol. Neurosurg. Psychiatry 23, 56-62 (1960).

25. Stroop, J. Studies of interference in serial verbal reactions. J. Exp. Psychol. 18 643-662 (1935).

26. Reitan, R. M. Validity of the trail making test as an indicator of organic brain damage. Percept. Mot. Skills 8, 271-276 (1958).

27. Folstein, M. F., Folstein, S. E. \& McHugh, P. R. Mini-mental state. A practical method for grading the cognitive state of patients for the clinician. J. Psychiatr. Res. 12, 189-198 (1975).

28. Anguera, J. A. et al. Video game training enhances cognitive control in older adults. Nat. Res. J. 501, 97-101 (2013).

29. Kroenke, K, Spitzer, R. L. \& Williams, J. B. The PHQ-9: validity of a brief depression severity measure. J. Gen. Intern. Med. 16, 606-613 (2001).

30. Eriksen, C. W. \& Schultz, D. W. Information processing in visual search: a continuous flow conception and experimental results. Percept. Psychophys. 25 249-263 (1979).

31. Forster, S. \& Lavie, N. Failures to ignore entirely irrelevant distractors: the role of load. J. Exp. Psychol. 14, 73-83 (2008)

32. Conners, C. K. et al. Conners' continuous performance Test II (CPT II v. 5). MultiHealth Syst. Inc. 29, 175-196 (2000).

33. Greenberg L. M., Kindschi C., Dupuy T., Hughes S. TOVA Clinical Manual. Los Alamitos, CA: TOVA Company; 2007.

34. Zhong, B. Single-arm phase IIA clinical trials with go/no-go decisions. Contemp. Clin. Trials 33, 1272-1279 (2012).

35. Chen, C. \& Beckman, R. A. Optimal cost-effective designs of Phase II proof of concept trials and associated go-no go decisions. J. Biopharmaceut. Stat. 19, 424-436 (2009).

36. Alexopoulos et al. Cognitive control, reward-related decision making and outcomes of late-life depression treated with an antidepressant. Psychol. Med. 45, 3111-3120 (2015).

37. Manning, K. J. et al. Executive functioning complaints and escitalopram treatment response in late-life depression. Am. J. Geriatr. Psychiatry $\mathbf{2 3}, \mathbf{4 4 0 - 4 4 5}$ (2015).

38. Pimontel, M. A. et al. A meta-analysis of executive dysfunction and antidepressant treatment response in late-life depression. Am. J. Geriatr. Psychiatn 24, 31-41 (2016).

39. Morimoto, S. S. et al. Executive function and short-term remission of geriatric depression: the role of semantic Strategy. Am. J. Geriatr. Psychiatry 19, 115-122 (2016).

40. Sneed, J. R. et al. Response inhibition predicts poor antidepressant treatment response in very old depressed patients. Am. J. Geriatr. Psychiatry 15, 553-563 (2007).

41. Fleming, T. M. et al. Serious games for the treatment or prevention of depression: a systematic review. Rev. Psicopatol. Psicol. Clín. 19, 16 (2015). 
42. Kim, S. M. et al. The effects of a serious game on depressive symptoms and anxiety in breast cancer patients with depression: a pilot study using functional magnetic resonance imaging. Games Health J. 7, 409-417 (2018).

43. Williams, L. M. et al. The ENGAGE study: Integrating neuroimaging, virtual reality and smartphone sensing to understand self-regulation for managing depression and obesity in a precision medicine model. Behav. Res Ther. 101 58-70 (2018).

44. Morimoto, S. S. et al. Targeting cognitive control deficits with neuroplasticitybased computerized cognitive remediation in patients with geriatric major depression: a randomized, double-blind, controlled trial. Am. J. Geriatr. Psychiatry 28, 971-980 (2020).
45. Koster, E. H. W., Hoorelbeke, K., Onraedt, T., Owens, M. \& Derakshan, N. Cognitive control interventions for depression: a systematic review of findings from training studies. Clin. Psychol. Rev. 53, 79-92 (2017).

46. Morimoto, S. S. et al. Executive dysfunction predicts treatment response to neuroplasticity-based computerized cognitive remediation (nCCR-GD) in elderly patients with major depression. Am. J. Geriatr. Psychiatry 24, 816-820 (2016).

47. Drysdale, A. T. et al. Resting-state connectivity biomarkers define neurophysiological subtypes of depression. Nat. Med. 23, 28-38 (2017) 